\title{
Detection of Ischemia in Patients with Asymptomatic Diabetes
}

\section{Deborah}

Associate Professor and Director, Adult Advanced Practice Nursing Specialty, Yale University School of Nursing
Individuals with both type 1 and type 2 diabetes mellitus (DM) are known to be at high risk for developing coronary artery disease (CAD). The risk is two to three times greater in those with DM compared with those without, and is especially high in women with DM. An additional problem in individuals with $\mathrm{DM}$ is that, even with severe underlying CAD, many individuals may have atypical symptoms or be totally asymptomatic, a condition referred to as silent or asymptomatic myocardial ischemia.

Asymptomatic myocardial ischemia is frequently found in individuals with known CAD, even in individuals without DM, and is associated with a poorer longterm prognosis. The Asymptomatic Cardiac Ischemia Pilot Study demonstrated that asymptomatic ischemia was associated with three times the risk of nonfatal myocardial infarction and six times the risk of myocardial infarction or death. ${ }^{1}$ However, asymptomatic myocardial infarction also occurs in a substantial number of individuals without known CAD, particularly those with DM. These individuals have not been diagnosed with CAD and do not have the benefit of anti-anginal therapies or aggressive CAD risk reduction. In the recent Detection of Ischemia in Asymptomatic Diabetics (DIAD) study, 21.6\% of older adults with type 2 DM, none of whom had any clinical evidence of CAD, had evidence of myocardial ischemia on single photon emission computed tomography (SPECT) myocardial perfusion imaging. ${ }^{2}$ This finding was consistent with earlier studies in the population with type $2 \mathrm{DM}^{3}$

In asymptomatic patients with either type 1 or type 2
DM, asymptomatic myocardial ischemia is also associated with major cardiac events. ${ }^{4}$ Importantly, the presence of cardiac autonomic neuropathy, long theorized to have a role in asymptomatic CAD, substantially increases the risk of subsequent cardiac events, with those individuals with both asymptomatic myocardial ischemia and cardiac autonomic neuropathy, being at highest risk. ${ }^{5}$

\section{Mechanisms-A Role For Cardiac Autonomic Neuropathy?}

Asymptomatic myocardial ischemia is often associated with cardiovascular autonomic neuropathy (CAN).This type of neuropathy affects the parasympathetic and sympathetic innervation of the heart and peripheral vasculature. It is frequently found in many individuals with DM, even at the time of initial DM diagnosis. The presence of CAN confers a greater risk of death.

The mechanisms responsible for CAD, as well as asymptomatic CAD, in individuals with DM are complex. Many factors including the presence of multiple known cardiac risk factors (hypertension, lipid abnormalities, obesity, microalbumiuria, and elevated homocysteine levels), hemostatic abnormalities, oxidative stress, inflammation, and hyperglycemia contribute to the development of CAD. Whether CAN is involved in the pathogenesis of CAD, or is merely a reflection of underlying disease is unclear. The reason for the silent nature of CAD in individuals with DM has long been debated; however, the presence of CAN has been consistently associated with asymptomatoic CAD. ${ }^{6}$

1. Stone P H, Chaitman B R, Forman $S$, et al,., "Prognostic significance of myocardial ischemia detected by ambulatory electrocardiography, exercise treadmill testing, and electrocardiogram at rest to predict cardiac events by one year (The Asymptomatic Cardiac Ischemia Pilot [ACIP] Study”, Am J Cardiol (1997);80: pp. 1,395-1,401.

2. Wackers F T H, Young L H, Inzucchi S E, et al. "Detection of silent myocardial ischemia in asymptomatic diabetic subjects: The DIAD Study”, Diabetes Care (2004);27: pp. 1,954-1,961.

3. Albers A R, Krichavsky m Z, Balady G J. "Stress testing in patients with diabetes mellitus", Circulation (2006);113: pp. 583-592.

4. Valensi P, Paries J, Brulport-Cerisier V, et al. "Predictive value of silent myocardial ischemia for cardiac events in diabetic patients: Influence of age in a French multicenter study”, Diabetes Care (2005); 28: pp. 2, 722-2, 727.

5. Valensi P, Sachs R-N, Harfouche B, et al. "Predictive value of cardiac autonomic neuropathy in diabetic patients with or without silent myocardial ischemia”, Diabetes Care (2001);24: pp. 339-343.

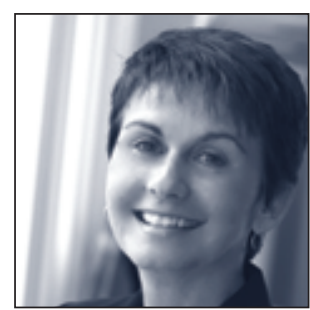

Deborah Chyun, RN, MSN, PhD, is Associate Professor and Director of the Adult Advanced Practice Nursing Specialty at Yale University School of Nursing. Dr Chyun is a Fellow of the American Heart Association (FAHA) and is also a member of the American Diabetes Association. She is a co-principal investigator in the Detection of Ischemia in Asymptomatic Diabetics (DIAD) study and is currently studying the impact of a multidisciplinary intervention in the type 2 diabetes population with or at high-risk of asymptomatic myocardial ischemia. 
The diagnosis of CAN is based on changes in heart rate (HR) during deep breathing (the expiratory/inspiratory ratio), standing, and Valsalva maneuvers, along with blood pressure responses to hand grip and standing. In addition, heart rate variability of $\mathrm{HR}$ interval changes (time-domain) or power spectral analysis of successive $\mathrm{R}-\mathrm{R}$ intervals (frequency-domain) are also commonly used to assess CAN. Recent recommendations are that all patients with type 2 DM be screened for CAN at the time of diagnosis and those with type 1 be screened five years after diagnosis. ${ }^{7}$ If screening is negative, it should be repeated yearly. CAN is important, not only because of its association with CAD and mortality, but also because it contributes to exercise intolerance and thus requires special precautions when individuals participate in physical activity.

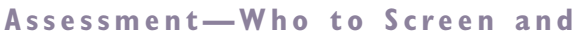

While the presence of CAN suggests that evidence of asymptomatic CAD should be sought, other clinical findings can also be used to decide who should undergo screening. However, our knowledge of who is at highest risk of asymptomatic CAD is limited by the fact that many previous studies have confined their study sample to include only those at high-risk of underlying CAD, while others have studied lower-risk populations, excluding individuals taking insulin or those unable to exercise adequately. Although it is recognized that neither traditional CAD risk factors, nor current consensus guidelines, adequately identify individuals at high-risk of asymptomatic CAD, ${ }^{2,8}$ several clinical factors may alert providers to its presence.

If CAD is suspected further assessment is required. The individual's level of physical activity should be carefully assessed, as well as the presence of any atypical symptoms such as extreme fatigue or anginal equivalents. When individuals want to engage in high intensity exercise, or in those with a long duration of diabetes, multiple CAD risk factors, or known DMrelated complications stress testing, using exercise or pharmacological stress, is often performed. ${ }^{9}$ Standard treadmill exercise electrocardiography (ECG) has limitations in the population with DM. The exercise ECG has a relatively low sensitivity, as well as yielding a significant number of false positive tests, especially in patients with hypertension and in women. In addition, many older patients with DM are unable to exercise adequately, decreasing the accuracy of the test, thus requiring the use of pharmacological agents (dobutamine or adenosine).

The addition of either echocardiography or myocardial perfusion imaging improves the sensitivity and specificity and provides additional useful prognostic information. During stress, echocardiography identifies regional wall motion abnormalities indicative of contractile dysfunction caused by ischemia. Myocardial perfusion imaging detects areas of diminished flow reserve in regions supplied by occluded arteries during stress. Large, reversible myocardial perfusion defects or severe ventricular dysfunction suggest significant areas of potentially jeopardized myocardium and these individuals should be referred for coronary angiography. Asymptomatic ischemia that has caused prior myocardial infarction, decreased left ventricular function, heart failure, or ventricular arrhythmias places these individuals at high-risk for subsequent cardiac events and therefore, these individuals require coronary angiography.

Newer techniques such as coronary artery calcium (CAC) imaging and ultrasound measurement of carotid intimal-media thickness (CIMT) provide evidence of atherosclerosis, rather than ischemia. Studies have demonstrated higher CAC scores (CCS) and increased CIMT in individuals with DM and that both are associated with poorer long-term outcomes. There is ongoing controversy as to whether all asymptomatic individuals with DM should be screened for CAD. The benefits of early detection of CAD might encourage more aggressive $\mathrm{CAD}$ risk reduction efforts on the part of both the individual and the provider, as well as ensure earlier percutaneous or surgical interventions. However, given the current knowledge gaps in regard to who is at highest risk, widespread screening is not believed to be cost effective. A recent review suggests that the presence of one additional CAD risk factor, an abnormal resting ECG, microalbuminuria or CAN should serve as an indication for CAD screening. ${ }^{10}$ As a first step, electron beam computer tomography (EBCT) for CAC or carotid ultrasound for CIMT should be performed. For CCS $<400$ or CIMT $<1 \mathrm{~mm}$ no further evaluation is

6. Vinik A I, Mitchell B D, Maser R E, Freeman R. "Diabetic autonomic neuropathy", Diabetes Care (2003);26: pp. 1,553-1,579.

7. Boulton A J M, Vinik A I, Arezzo J C, et al. "Diabetic neuropathies: A statement by the American Diabetes Association", Diabetes Care (2005);28: pp. 956-962.

8. American Diabetes Association. "Standards of medical care in diabetes", Diabetes Care (2006);suppl 1: pp. S4-S42.

9. American Diabetes Association. "Consensus development conference on the diagnosis of coronary heart disease in people with diabetes”, Diabetes Care (1998);21: pp. 1,551-1,559. 
suggested, while for levels above these cutpoints, echocardiography or stress SPECT should be used to determine the additional need for coronary angiography.

\section{Nursing Implications}

Nursing has a pivotal role in the prevention, early detection and management of asymptomatic CAD. As all individuals with DM are at high-risk of both symptomatic and asymptomatic CAD, multifactorial risk reduction, including intensive control of glucose, weight, blood pressure, and lipid levels, as well as smoking cessation and increasing levels of physical activity is a crucial component of DM education. Identification of patients at high-risk of asymptomatic myocardial ischemia, including those with CAN, may assist in the early detection of asymptomatic ischemia prior to irreversible myocardial injury.
CAD may result in anxiety, depression and psychological distress, all of which may hamper the individual's self-management ability and adversely affect their quality of life. Screening, on the other hand may have some psychological benefit. As in all aspects of DM management, close patient-provider communication about the meaning of the screening results and ongoing CAD risk reduction is necessary.

In addition, the identification of asymptomatic CAD has important implications. Not only does it reinforce the need to aggressively reduce modifiable CAD risk factors, including perhaps addition of multiple medications for DM, blood pressure, and lipid control, as well as lifestyle modification, but may also demand additional testing and subsequent interventions for which the individual should be prepared. If CAD is present then daily aspirin is usually added and beta-

\section{Identification of patients at high-risk of asymptomatic myocardial ischemia, including those with $C A N$, may assist in the early detection of asymptomatic ischemia prior to irreversible myocardial injury.}

Many individuals with DM are unaware of the problem of asymptomatic CAD and CAN, being more familiar with microvascular complicationsretinopathy, nephropathy, or painful peripheral neuropathy. Education regarding the signs or symptoms of other forms of autonomic neuropathy, as well as CAN should be provided, along with stressing the importance of glucose and CAD risk factor control as a means to prevent these complications. Individuals with DM should also be aware that they are at an increased risk of developing CAD and of typical and atypical signs of symptoms, including the fact that women and older individuals, as well as people with DM tend to present atypically. They should also be instructed how to respond and how soon to seek help if symptoms do not resolve on their own.

Little is known regarding the behavioral and emotional impact of screening for asymptomatic CAD ${ }^{11}$ Negative results, even in the presence of an adverse risk factor profile, may lead to the mistaken belief that one does not have to engage in lifestyle changes. Detection of blockers may also be prescribed to prevent ischemia. Recommendations for regular exercise should be reinforced with limitations placed on strenuous activity that might place the patient at risk for a cardiac event. The complete absence of angina or equivalents may make it difficult for the individual to gauge activity, as well as contribute to psychological distress.

The problem of asymptomatic myocardial ischemia in individuals with DM is significant. As the prevalence of DM continues to increase, particularly in youth and younger-aged adults, it is likely to take on increased importance. The need for a better understanding of the pathogenesis and management of asymptomatic disease is required, as is as is identification of high-risk individuals. In addition, a well-tested strategy for screening for CAD is urgently needed, along with a better appreciation of the psychological and behavioral sequelae from both positive and negative test results. Longitudinal follow-up from screening studies such as the DIAD Study, expected in 2007, will also assist in determining the benefits of screening in this high-risk population.

10. Raggi P, Bellasi A, Ratti C. "Ischemia imaging and plaque imaging in diabetes", Diabetes Care (2005);28: pp. 2787-2,794.

11. Chyun D A, Katten D M, Melkus G, Talley S, Davey J A, Wackers FJTh. "The impact of screening for asymptomatic myocardial ischemia in individuals with type 2 diabetes”, J CV Nursing (2006); 21; pp. E1-E7. 